\title{
Solar Tracking Application
}

\author{
Soumya Ranjita Nayak ${ }^{1}$, Chinmaya Ranjan Pradhan ${ }^{2}$ \\ ${ }^{1}$ (Asst.prof in Electrical and Electronics Engineering, BRMIIT, Bhubaneswar, Odisha, India) \\ ${ }^{2}$ (Asst.prof in Electrical and Electronics Engineering, BRMIIT, Bhubaneswar, Odisha, India)
}

\begin{abstract}
The increasing demand for energy, the continuous reduction in existing sources of fossil fuels and the growing concern regarding environment pollution, have pushed mankind to explore new technologies for the production of electrical energy using clean, renewable sources, such as solar energy, wind energy, etc. Among the non-conventional, renewable energy sources, solar energy affords great potential for conversion into electric power, able to ensure an important part of the electrical energy needs of the planet.Solar energy is rapidly gaining notoriety as an important means of expanding renewable energy resources. More energy is produced by tracking the solar panel to remain aligned to the sun at a right angle to the rays of light. This paper deals with application of solar trackers.
\end{abstract}

Keywords - Solar Tracker, Single Axis, Dual Axis, Tracker Component, Sun Tracking algorithm

\section{INTRODUCTION}

Solar trackers are devices used to orient photovoltaic panels, reflectors, lenses or other optical devices toward the sun. Since the sun's position in the sky changes with the seasons and the time of day, trackers are used to align the collection system to maximize energy production. Several factors must be considered when determining the use of trackers. Some of these include: the solar technology being used, the amount of direct solar irradiation, feed-in tariffs in the region where the system is deployed, and the cost to install and maintain the trackers. Concentrated applications like concentrated photovoltaic panels (CPV) or concentrated solar power (CSP) require a high degree of accuracy to ensure the sunlight is directed precisely at the focal point of the reflector or lens. Non-concentrating applications don't require tracking but using a tracker can improve the total power produced by the system. Photovoltaic systems using high efficiency panels with trackers can be very effective.

There are many types of solar trackers, of varying costs, sophistication, and performance. The two basic categories of trackers are single axis and dual axis.

\section{SINGLE AXIS}

Solar trackers can either have a horizontal or a vertical axis. The horizontal type is used in tropical regions where the sun gets very high at noon, but the days are short. The vertical type is used in high latitudes where the sun does not get very high, but summer days can be very long. In concentrated solar power applications, single axis trackers are used with parabolic and linear Fresnel mirror designs.

\section{DUAL AXIS}

Solar trackers have both a horizontal and a vertical axis and thus they can track the sun's apparent motion virtually anywhere in the world. CSP applications using dual axis tracking include solar power towers and dish (Stirling engine) systems. Dual axis tracking is extremely important in solar tower applications due to the angle errors resulting from longer distances between the mirror and the central receiver located in the tower structure.

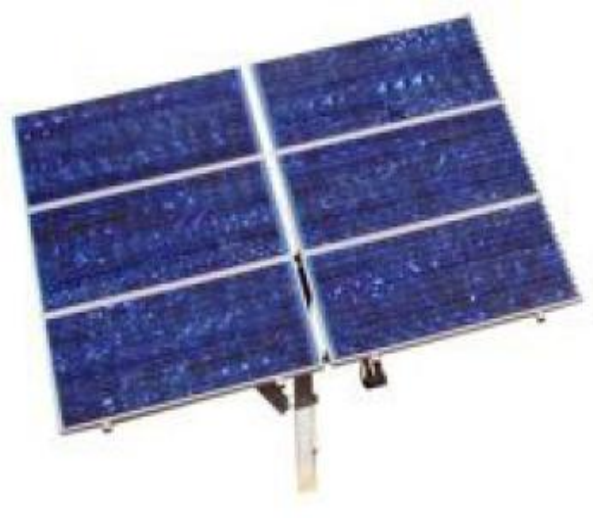

[SINGLE AXIS SOLAR TRACKER]

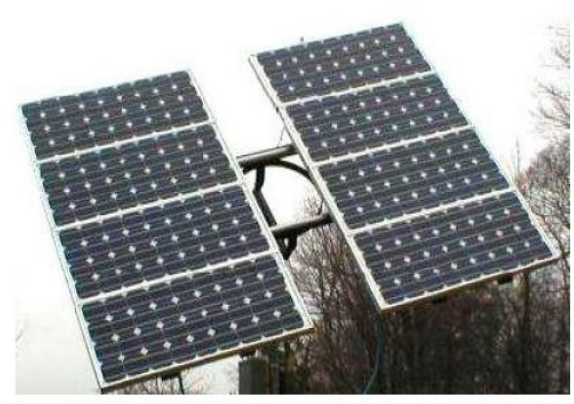

[DUAL AXIS SOLAR TRACKER] 


\section{TRACKER COMPONENTS}

The main elements of a tracking system are as follows:

1. Sun tracking algorithm: This algorithm calculates the solar azimuth and zenith angles of the sun. These angles are then used to position the solar panel or reflector to point toward the sun. Some algorithms are purely mathematical based on astronomical references while others utilize real-time light-intensity readings.

2. Control unit: The control unit executes the sun tracking algorithm and coordinates the movement of the positioning system.

3. Positioning system: The positioning system moves the panel or reflector to face the sun at the optimum angles. Some positioning systems are electrical and some are hydraulic. Electrical systems utilize encoders and variable frequency drives or linear actuators to monitor the current position of the panel and move to desired positions.

4. Drive mechanism/transmission: The drive mechanisms include linear actuators, linear drives, hydraulic cylinders, swivel drives, worm gears, planetary gears, and threaded spindles.

5. Sensing devices: For trackers that use light intensity in the tracking algorithm, pyranometers are needed to read the light intensity. Ambient condition monitoring for pressure, temperature and humidity may also be needed to optimize efficiency and power output.

I. Limit switches are used to control speed and prevent over travels. The mechanical over travel limits are used to prevent tracker damage.

II. Elevation feedback is accomplished by either 1) a combination of limit switches and motor encoder counts, or 2) an inclinometer (a sensor that provides the tilt angle).

III. An anemometer is used to measure wind speed. If the wind conditions are too strong, the panels are usually driven to a safe horizontal position and remain in the safety position until the wind speed falls below the set point.

\section{TRACKER CONTROL ALGORITHMS}

Tracker control algorithms typically incorporate a control strategy that is a hybrid between open-loop and closed loop control. The open-loop component is needed because the sun can be obscured by clouds, eliminating or distorting the feedback signals. The closed-loop component is needed to eliminate errors that result from variability in installation, assembly, calibration, and encoder mounting. Closed loop systems track the sun by relying on a set of lenses or sensors with a limited field of view, directed at the sun, and are fully illuminated by sunlight at all times. As the sun moves, it begins to shade one or more sensors, which the system detects and activates motors or actuators to move the device back into a position where all sensors are once again equally illuminated.

Open loop systems track the sun without physically following the sun via sensors (although sensors may be used for calibration). These systems typically employ electronic logic which controls device motors or actuators to follow the sun based on a mathematical formula.

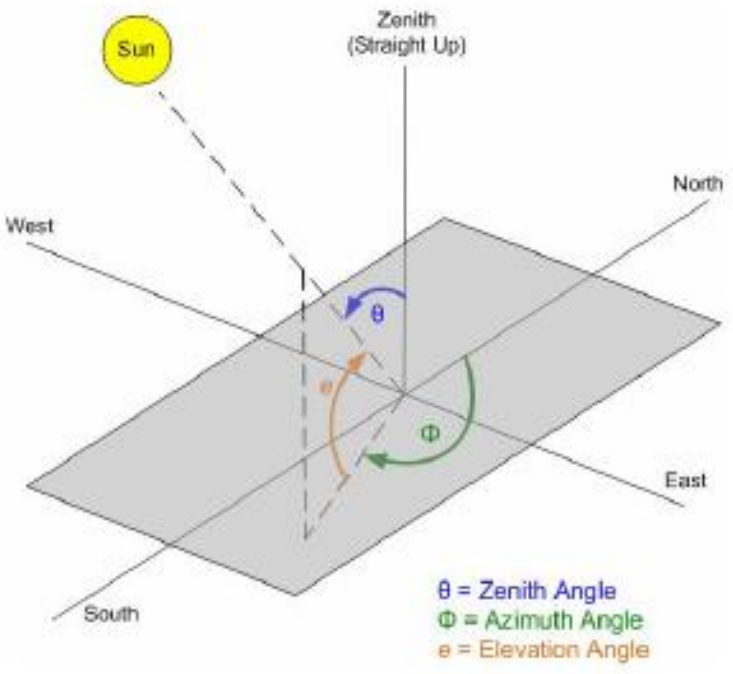

The Zenith angle is the angle between the direction of the sun (direction of interest) and the zenith (straight up or directly overhead). The program calculates the Zenith angle in degrees and stores it in register SPA_ZENITH. This would be the TILT angle with $0^{\circ}$ being horizontal. The suns elevation or altitude is the angle from the horizontal plane and the Suns central ray or just the compliment of the Zenith angle $\left(90^{\circ}\right.$ - Zenith angle).

The Azimuth angle is measured clockwise from true north to the point on the horizon directly below the object. The program calculates the local (from observer) azimuth angle (eastward from North) [0 to 360 degrees] and stores it in register SPA_AZIMUTH. This would be the ROTATION angle with $0^{\circ}$ facing north.

\section{SOLUTION}

MicroLogix controller helps position the solar panels, mirrors, or lenses into the sun in order to capture the sun's energy. The MicroLogix controller can be equipped with a positioning algorithm to mathematically solve the optimum tracker position for any time of the day. The outputs of the calculation are two angles - the zenith angle and the azimuth angle. The azimuth angle is used to determine the horizontal rotation and the zenith angle is used to determine the vertical tilt or elevation. Depending on the type of tracker being used (single or dual axis), one or both of these angles are then used to position the solar collection device. The position 
loop can be closed in the controller or in the drive if the axis is so equipped. If the controller is used, the embedded High Speed Counter (HSC) instruction and corresponding inputs on the MicroLogix controller are utilized. Utilizing a drive that accepts an external encoder allows the position loop to be managed within the drive.

If drives are utilized, there are a few control capabilities that must be considered. They include being able to shut down the drives at night or long periods of inactivity to save energy. During shutdown periods, the position must be maintained or the tracker must go through a homing cycle before beginning the new day's operation. A motor brake could be used if a homing cycle is not permitted. At the end of each day, the tracker will need to return to the starting or sunrise position.

In non-concentrating applications, linear actuators offer an alternative method to control a single axis solar tracker or the elevation in a dual axis solar tracker. Positioning the panel within a few degrees is often acceptable so there are less move intervals over the course of the day.

Automatic emailing from the tracker controller to operators or maintenance personnel can alert them of tracker problems. Maintenance costs can be reduced by being able to remotely adjust the tracker and to monitor tracker operation and status. When many trackers are installed and managed in a solar field, a central controller is often required to coordinate the trackers and monitor them. An alternative to running the positioning algorithm in each tracker controller would be to utilize this central controller to execute the mathematical calculations and communicate the desired position coordinates to the respective trackers.

\section{SOLUTION BENEFITS}

\section{High Precision Sun Tracking Algorithm}

Following the NREL Solar Position Algorithm for Solar Radiation Applications Technical Report, positioning with $+/-0.005^{\circ}$ accuracy using the MicroLogix platform can be achieved.

\section{Calculated Angles}

The derived angles can be applied in solutions using hydraulic or electrical positioning methods.

3. High quality, Industrial Hardened component Controllers built to industry standards (UL, IEC, CE, etc.) lowers installation and maintenance costs over "black box" controllers for harsh industrial applications.

\section{Off-the-Shelf (OTS) Components}

Globally available OTS components are supported worldwide by a network of industry technical specialists, distributors, and systems integrators. OTS components reduce time to market for the OEM and meet end user goals for global support.

\section{Supply Chain Solutions}

As a trusted supply chain partner, Rockwell Automation offers a global manufacturing and distribution network including panel design and fabrication capabilities worldwide. Panels can be fully tested and delivered to the site for quick installation.

6. Secure Remote Access/Monitoring

Remote monitoring allows OEMs and end users to adjust trackers, diagnose problems and monitor operations reducing operation and maintenance costs.

7. Fast, Efficient Data Communications

Large solar field deployments can utilize open EtherNet/IP networks to achieve high speed, large data transfers (coordinated moves for multiple trackers) from a main control system to the individual trackers.

\section{Faster Development/Troubleshooting}

Pre-programmed and tested positioning algorithm logic; Human Machine Interface (HMI) faceplates provide detailed networking diagnostics, drive operating parameters and status.

9. Product Life Cycle

Rockwell Automation's 100+ year history of providing quality products and services helps users with the support longevity they need to operate their solar farm. Rockwell's commitment to long product life cycles and well defined migration paths provides the peace of mind that the products selected today will be supported for years to come. 


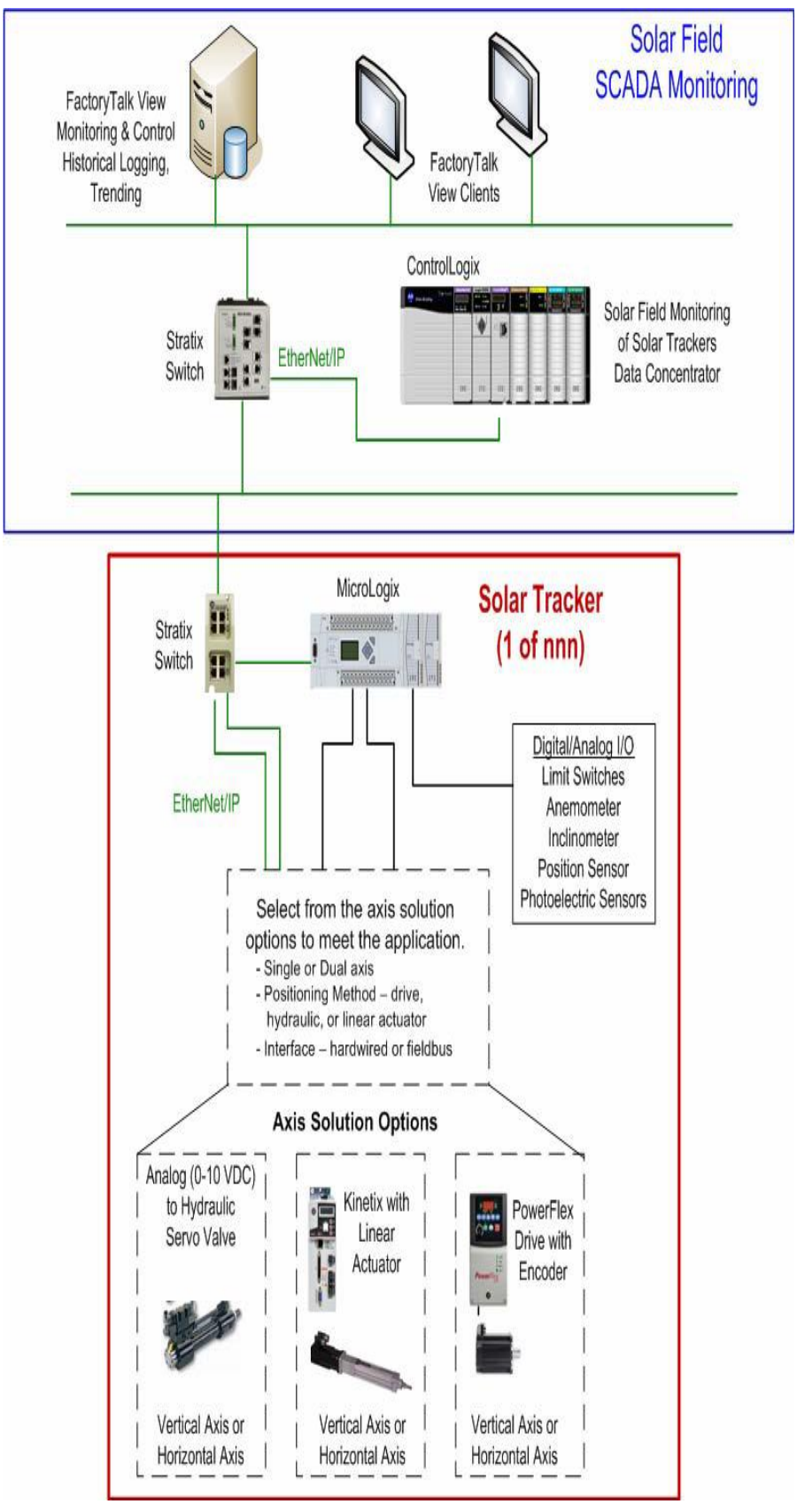

VII. CONCLUSION

Rockwell Automation offers several solutions to help energy providers generate the most solar power from their system. The Rockwell Automation solution for solar trackers allows machine builders to provide an off-the- shelf solution to both single and dual axis tracking solutions. The code template provided for calculating angles needed for high accuracy tracking reduces development time and allows machine builders to focus their attention on the positioning system rather than the mathematical equations.

\section{ACKNOWLEDGEMENTS}

For this research, The authors would also like to thank to Prof.(Dr) S.M.Ali,School of Electrical Engineering,KIIT
University for his support and valuable contributions towards the success of this research.

\section{REFERENCES}

[1] Mohd.Azab, 2010. Optimal Power point tracking for StandAlone PV System using Particle Swarm Optimization. IEEE Symposium on Industrial Electronics (ISIE), July 2010, pp. 969- 973.

[2] AyuWaziraAzahari,KamaruzzamanSopian, AzamiZaharim and Mohd Al Ghoul, 2008. A New Approach for Predicting Solar Radiation in Tropical Environment using Satellite Images-Case Study of Malaysia. WSEAS Transactions on Environment and Development, Issue 4 Vol.4, April 2008, pp. 373-378.

[3] M.Mohandes, A.Balghonaim, M.Kassas, S.Rehman and O.Halawani, 2000. Use Radial Basis Function for Estimating Monthly Mean Daily Solar Radiation. Elsevier Science: Solar Energy Vol.68, No.2, pp. 161- 168.

[4] Momoh, J.A. , Wang,Y. , and Eddy-Posey, F. , Optimal Power Dispatch of Photovoltaic System with Random Load. Power Engineering Society General Meeting, IEEE, 2004 1010 June 2004, Vol. 2, pp. 1939-1945.

[5] Atsu S.S. Dorlo, Joseph A. Jervase, Ali Al-Lawati, 2002. Solar Radiation Estimation using Artificial Neural Networks. Elsevier Science: Applied Energy, pp. 307-319.

[6] Joe-Air Jiang, Tsong-Liang Huang, Ying-Tung Hsiao and Chia- Hong Chen, 2005. Maximum Power Tracking for Photovoltaic Power System. Tamkang Journal of Science and Engineering, 2005, Vol. 8, No 2, pp. 147-153.

[7] Ramaprabha, R., Gothandaraman, V., Kanimozhi, K., Divya, R., Mathur, B.L. Maximum power point tracking using GA optimizedartificial neural network for Solar PV system, Electrical Energy Systems (ICEES), 2011 1st International Conference on, 3-5 Jan 2011, pp.264-268.

[8] Xiaojin Wu, Xueye Wei, Tao Xie, Rongrong Yu, 2010. Optimal Design of Structures of PV Array in Photovoltaic Systems, Intelligent System Design and Engineering Application (ISDEA), 2010 International Conference on , vol.2, no., pp.9-12, 13-14 Oct. 2010.

[9] Adel Mellit, S.Shaari, H.Mekki and N.Khorissi, 2008. FPGA based Artificial Neural Network for Prediction of Solar Radiation Data from Sunshine Duration and Air Temperature. Region 8 Sibircon, IEEE, 2008, pp. 118-123.

[10] Francisco M. and González-Longatt, 2005. Model of Photovoltaic Module in MATLAB. 2nd American Student Congress of Electrical, Electronics and Computer Engineering (II CIBELEC), 2005, pp. 1-5.

[11] A.K. Saxena and V. Dutta, "A versatile microprocessor based controller for solar tracking," in Proc. IEEE, 1990, pp. 1105 $-1109$.

[12] S. J. Hamilton, "Sun-tracking solar cell array system," Department ofComputer Science and Electrical Engineering, University of Queensland, Bachelors Thesis, 1999.

[13] Tracking Mode to Gain High Concentration Solar Energy", IEEE Conference, 12-14April 2007, Page(s):551 - 555.

[14] [Pritchard, 1983] Daniel A. Pritchard, "Sun Tracking by Peak Power Positioning for Photovoltaic Concentrator Arrays" IEEE Transactions on Control System, Volume3, Issue3, Aug1983,Page(s):2-8. 